\title{
Extremal problems related to convexity
}

\author{
Aimo Hinkkanen ${ }^{1}$ and Sineenuch Suwannaphichat ${ }^{2 *}$ (B)
}

${ }^{*}$ Correspondence:

suwannaphichat_s@silpakorn.edu

${ }^{2}$ Department of Mathematics,

Faculty of Science, Silpakorn

University, Nakhon Pathom, 73000,

Thailand

Full list of author information is

available at the end of the article

\begin{abstract}
We consider the extremal problem of maximizing functions $u$ in the class of real-valued biconvex functions satisfying a boundary condition $\psi$ on a product of the unit ball with itself, with the $\ell^{p}$-norm. In 1986, Burkholder explicitly found the maximal function for $p=2$. In this paper, we find some characterizations of such extremal functions. We establish that sufficiently smooth solutions to the convex extremal problems with given boundary values are affine on line segments and the domain $D$ is foliated by such segments.
\end{abstract}

Keywords: convex; extremal function; affine

\section{Introduction}

In this paper, we consider properties of convex functions defined in a convex plane domain $D$ that are maximal with respect to prescribed real boundary values. We show that if such an extremal function is in $C^{3}$, then it is affine on certain line segments that foliate the domain $D$. This structural result represents a very preliminary step in a larger program of determining maximal $\zeta$-convex functions in certain Banach spaces.

For precise definitions and background results we refer to Section 3. But let us note here that in Banach space theory one considers Banach spaces that are $\zeta$-convex, that is, they support a biconvex function $\zeta$ satisfying (6). The question arises as to which Banach spaces are $\zeta$-convex, and for them, what the largest biconvex function satisfying (6) is equal to. Banach spaces of the type $\ell^{p}$ and $L^{p}$ are known to be $\zeta$-convex for $1<p<\infty$. However, the extremal function $\zeta$ has been determined only for Hilbert spaces, by Burkholder in 1986. In 2008, Saksman drew the attention of the first author to the fact that the extremal function is not known in $\ell^{p}$ - and $L^{p}$-spaces for $p \neq 2$, even though it might be desirable to know it. This question provides the basic motivation for the present paper.

In order to get started with the study of such a question, we only consider the simplest non-trivial case, that of the Banach space $\ell^{p}$ in $\mathbb{R}^{2}$. Any results obtained in this case might then have generalizations to other cases. Let $\bar{B}$ be the closed unit ball in $\mathbb{R}^{2}$ in the $\ell^{p}$ metric. We are looking for the largest biconvex function $\zeta: \bar{B} \times \bar{B} \rightarrow \mathbb{R}$ such that $\zeta(x, y) \leq$ $|x+y|_{p}$ whenever $|x|_{p}=|y|_{p}=1$. The Hilbert space case suggests that not much is lost if one fixes one variable, say $y$, and only uses initially the assumption that $x \rightarrow \zeta(x, y)$ is a convex function of $x \in \bar{B}$. In this spirit we consider the slightly more general problem of finding or characterizing the maximal function $u: D \rightarrow \mathbb{R}$ defined in the closure of a bounded convex plane domain, such that $u(x) \leq \psi(x)$ for all $x \in \partial D$, for a given function $\psi$ on $\partial D$. If the boundary function $\psi$ is not sufficiently regular, it may of course happen that $\psi_{0}(x)=u(x)$

(c) The Author(s) 2016. This article is distributed under the terms of the Creative Commons Attribution 4.0 International License (http://creativecommons.org/licenses/by/4.0/), which permits unrestricted use, distribution, and reproduction in any medium, provided you give appropriate credit to the original author(s) and the source, provide a link to the Creative Commons license, and indicate if changes were made. 
for $x \in \partial D$, for the extremal function $u$ satisfies only $\psi_{0} \leq \psi$ but not necessarily $\psi_{0}=\psi$ on $\partial D$. However, the same extremal function $u$ is then obtained for boundary values $\psi_{0}$ and the structural results that we obtain allow us to express $u$ in the interior of $D$ in terms of $\psi_{0}$ (which, in interesting applications, will often be the same as $\psi$ ) as soon as we know a certain foliation of $D$.

In applications it is likely that the extremal function is many times differentiable, perhaps even in $C^{\infty}$, in the interior of $D$. Thus we see no significant loss of generality in assuming, in this paper, that $u$ is in $C^{3}$ and pursuing the implications of this assumption.

We first observe that the extremal function must be a solution of the Hopf differential equation. Indeed, we find that with $x=\left(x_{1}, x_{2}\right) \in D$, the function $A=u_{x_{1} x_{1}} / u_{x_{1} x_{2}}=$ $u_{x_{1} x_{2}} / u_{x_{2} x_{2}}$ satisfies such an equation. The general form of such solutions is known, and they are given implicitly in terms of a parameter function $\Phi$. We then ask what can be said about such functions $A$ and, consequently, about the function $u$. We find that at each point $x$ of $D$, the value of $A(x)$ determines a line $L$ through $x$ and hence the line segment $L_{1}=L \cap D$. We show that $A$ is constant and that $u$ is affine on each such segment $L_{1}$. This shows that the domain $D$ is foliated by such line segments: the segments are disjoint and their union is $D$. Hence on each segment $L_{1}$, the function $u$, being affine, is determined by its values at the end points of $L_{1}$, which are on $\partial D$, and these boundary values of $u$ are the values of $\psi_{0}$, hence often the same as the values of $\psi$.

Thus, if we knew what the foliation is explicitly, we could then compute the extremal function $u$. However, at this stage many foliations are still possible, and not all of them correspond to the extremal function. Further work shows that one can develop a differential equation for a parametrization of the foliation, and the solution of that equation will give the sought-for extremal function. The equation is complicated and we will leave the presentation of this work to another paper. When $p=2$, we recover the extremal function $u$ discovered by Burkholder. When $p \neq 2$, the equation is complicated and it is not clear if explicit solutions can be found.

However, the structural results of this paper are of interest in their own right. In various papers of Burkholder, it has been pointed out that certain functions discovered there are affine on certain line segments, apparently without the realization that this is a general property of this type of extremal functions. Thus we gain a greater understanding of the nature of the solutions to these extremal problems. For example, in [1], p.687, (9.7), it is observed that a certain extremal biconcave function is affine on certain line segments. Similarly, Burkholder has noted that his extremal function (7) is affine on certain line segments. We now see that this is to be expected and that one can further approach all such problems by specifically looking for such foliations.

On the basis of preliminary studies, we feel that it is likely that our methods will work more generally in $\mathbb{R}^{n}$, for all $n \geq 2$, and it may be that similar results are valid in more general Banach spaces. This work will have to be the subject of a later paper.

It seems to be typical in applications that in fact, the convex domain $D$ is divided by a line segment into two parts, in each of which the function $A$ described above is well defined. Thus there is a foliation in each of the two parts of $D$ and the separating segment does not belong to either foliation (or, in an extended sense, belongs to both). This is connected to certain second order partial derivatives of $u$ being zero on the separating segment. The assumptions of our theorems exclude this case, but the phenomena described in the theorems of this paper are then valid in each of the two parts of $D$, each of them being a convex 
domain in its own right. When considering each part as a convex domain, the boundary values are not known on that part of the boundary corresponding to the separating segment, but this does not matter as that segment will not be involved in the foliation of the interior of either part of $D$.

\section{Results}

In Hilbert space, the greatest biconvex function in a class of biconvex functions satisfying certain boundary conditions was explicitly found by Burkholder [2] in 1986, see Theorem 3.1 in Section 3. Such a result would imply $\zeta$-convexity which is one of the three equivalent conditions giving some geometric characterizations of Banach spaces, see [3]:

$\zeta$-convex $\Longleftrightarrow \mathrm{UMD} \Longleftrightarrow$ HT-space.

To study the extremal problems further in general Banach spaces, we may investigate the characterizations of such extremal functions. We consider the problem of finding the extremal function in the class of real-valued biconvex functions $u$ satisfying a boundary condition $\psi$ on a domain $D \times D$ where $D$ is a convex domain in $\mathbb{R}^{2}$. In particular, we restrict the domain $D$ to the unit ball in the $\ell^{p}$-norm or the unit disk when $p=2$. We establish that sufficiently smooth solutions to the convex extremal problems with given boundary values are affine on line segments and the domain $D$ is foliated by such segments.

In $\ell^{p}\left(\mathbb{R}^{2}\right)$, we denote the $\ell^{p}$-norm by $|x|_{p}$ where $x=\left(x_{1}, x_{2}\right)$ in $\mathbb{R}^{2}$. Thus $|x|_{p}^{p}=\left|x_{1}\right|^{p}+\left|x_{2}\right|^{p}$. We define the open and closed unit balls in $\mathbb{R}^{2}$ for the $\ell^{p}$-metric by $B=\left\{x \in \mathbb{R}^{2}:|x|_{p}<1\right\}$ and $\bar{B}=\left\{x \in \mathbb{R}^{2}:|x|_{p} \leq 1\right\}$.

Let $u: \bar{B} \times \bar{B} \rightarrow \mathbb{R}$ be a continuous function that is in $C^{3}$ in $B \times B$. Fix $y=\left(y_{1}, y_{2}\right) \in \bar{B}$. Then we can consider $u$ as a function of $x_{1}$ and $x_{2}$, where $x=\left(x_{1}, x_{2}\right)$ in $\bar{B}$. For $x_{1}=\cos \theta$, $x_{2}=\sigma(\theta)= \pm\left(1-|\cos (\theta)|^{p}\right)^{1 / p}$ and $y=\left(y_{1}, y_{2}\right) \in \bar{B}$, we define a boundary function $\psi$ by

$$
\psi(\theta)=u(\cos (\theta), \sigma(\theta))=\left(\left|\cos (\theta)+y_{1}\right|^{p}+\left|\sigma(\theta)+y_{2}\right|^{p}\right)^{1 / p}=|x+y|_{p}
$$

When $p=2$, this function $\psi$ is the same boundary function as in Burkholder's result [2].

We denote the partial derivatives of a function $u=u\left(x_{1}, x_{2}\right)$ by using subscripts, for example,

$$
u_{x_{1} x_{2}}=\frac{\partial^{2} u}{\partial x_{1} \partial x_{2}} .
$$

We define a real-valued function $A$ on $\bar{B}$ by

$$
A\left(x_{1}, x_{2}\right)=\frac{u_{x_{1} x_{1}}}{u_{x_{1} x_{2}}}=\frac{u_{x_{1} x_{2}}}{u_{x_{2} x_{2}}},
$$

assuming that $u_{x_{1} x_{1}}, u_{x_{2} x_{2}}$ and $u_{x_{1} x_{2}}$ do not vanish there (a milder requirement would be that $u_{x_{1} x_{1}}, u_{x_{2} x_{2}}$ and $u_{x_{1} x_{2}}$ do not vanish on any open set, but we do not address that here). If $u \in C^{3}$, then differentiation shows that we have the relation

$$
A A_{x_{2}}=A_{x_{1}}
$$


which is a special case of the Hopf differential equation. Hence, the general solution $A=$ $A\left(x_{1}, x_{2}\right)$ of this equation is given implicitly by

$$
\Phi(A)=x_{2}+A x_{1}
$$

where $\Phi$ is a suitable real-valued function (see [4]). Different admissible choices for $\Phi$ give rise to different solutions $A$.

From this perspective and using these notations, we obtain the following theorems.

Theorem 2.1 Let $A=A\left(x_{1}, x_{2}\right)$ be a continuous real-valued function defined for $\left(x_{1}, x_{2}\right) \in$ $D$, where $D$ is the closure of a bounded convex plane domain. If $\Phi$ is a continuous real-valued function on an interval of the real axis that contains the set $A(D)$, and if $\Phi\left(A\left(x_{1}, x_{2}\right)\right)=x_{2}+x_{1} A\left(x_{1}, x_{2}\right)$ in $D$, then $A\left(x_{1}, x_{2}\right)$ is constant on certain line segments that are maximal in the sense that each segment is the intersection of $D$ with a straight line. The union of such line segments is $D$. Moreover, if the real-valued function $u$ is in $C^{2}$ in $D$ and satisfies (2) there (which, in particular, means that $u_{x_{2} x_{2}}$ and $u_{x_{1} x_{2}}$ do not vanish in D), then $u$ is affine on each such segment.

Remark 2.2 When $D=\bar{B}$, Theorem 2.1 gives a foliation of the entire domain $\bar{B}$.

Theorem 2.3 Let u be a convex $C^{2}$ real-valued function on a bounded convex plane domain $D$. If $u$ is affine on an open line segment $L$ in $D$, then at each point of $L$ we have

$$
u_{x_{1} x_{1}} u_{x_{2} x_{2}}-u_{x_{1} x_{2}}^{2}=0 \text {. }
$$

Furthermore, at each point of $L$ where $u_{x_{1} x_{2}} \neq 0$ and $u_{x_{2} x_{2}} \neq 0$, we have

$$
\frac{u_{x_{1} x_{1}}}{u_{x_{1} x_{2}}}=\frac{u_{x_{1} x_{2}}}{u_{x_{2} x_{2}}}
$$

Let now $D$ be the closure of a bounded convex plane domain, let $\psi$ be a continuous realvalued function on $\partial D$, and let $\mathcal{F}$ be the set of real-valued convex functions $v$ on $D$ such that $v \leq \psi$ on $\partial D$. Define

$$
u(x)=\sup \{v(x): v \in \mathcal{F}\} .
$$

Following Burkholder, we see that $u$ is convex and $u \in \mathcal{F}$, so that $u$ is the maximal function in $\mathcal{F}$. Suppose that $u$ is in $C^{3}$ in the interior of $D$. The convexity of $u$ implies that at each point we have $u_{x_{1} x_{1}} \geq 0, u_{x_{2} x_{2}} \geq 0$, and $u_{x_{1} x_{1}} u_{x_{2} x_{2}}-u_{x_{1} x_{2}}^{2} \geq 0$. As noted by Burkholder, at each point equality must hold in at least one of these inequalities, for otherwise we may modify $u$ slightly in a small neighborhood of the point and get an even larger element of $\mathcal{F}$. Now if $u_{x_{1} x_{1}}=0$ or $u_{x_{2} x_{2}}=0$, then from

$$
u_{x_{1} x_{1}} u_{x_{2} x_{2}}-u_{x_{1} x_{2}}^{2}=-u_{x_{1} x_{2}}^{2} \geq 0
$$

we deduce that $u_{x_{1} x_{2}}=0$, so that

$$
u_{x_{1} x_{1}} u_{x_{2} x_{2}}-u_{x_{1} x_{2}}^{2}=0 \text {. }
$$

Thus (4) holds at each point of the interior of $D$. 
If we now assume that $u_{x_{1} x_{2}} \neq 0$ and $u_{x_{2} x_{2}} \neq 0$ in $D$, it follows that we may define the function $A$ as in (2), and then $A$ is given by (3) for a suitable function $\Phi$. Now from Theorem 2.1 we see that $u$ is affine on line segments that foliate $D$. Thus we obtain the following result.

Theorem 2.4 Let D be the closure of a bounded convex plane domain, let $\psi$ be a continuous real-valued function on $\partial D$, and let $u$ be the maximal real-valued convex function on $D$ such that $u \leq \psi$ on $\partial D$. Suppose that $u \in C^{3}$. Then (4) holds at each point of the interior of $D$. Furthermore, if $u_{x_{1} x_{2}} \neq 0$ and $u_{x_{2} x_{2}} \neq 0$ in $D$, then we may define the function $A$ as in (2), and then $A$ is given by (3) for a suitable function $\Phi$. Finally, $u$ is affine on line segments that foliate $D$.

We conclude with the remark that the property of $u$ being affine on line segments is equivalent to (4) in a suitable sense.

Theorem 2.5 Let D be a bounded convex plane domain. Let $u: D \rightarrow \mathbb{R}$ be a convex function in the class $C^{3}$. Then, on certain line segments that are maximal in the sense that each segment is the intersection of $D$ with a straight line, we have

$$
\frac{u_{x_{1} x_{1}}}{u_{x_{1} x_{2}}}=\frac{u_{x_{1} x_{2}}}{u_{x_{2} x_{2}}} \text { if, and only if, } u \text { is affine on the line segment, }
$$

assuming that $u_{x_{1} x_{2}} \neq 0$ and $u_{x_{2} x_{2}} \neq 0$.

Namely, if $u$ is affine on line segments as stated, then it follows from Theorem 2.3 that $u_{x_{1} x_{1}} / u_{x_{1} x_{2}}=u_{x_{1} x_{2}} / u_{x_{2} x_{2}}$ on these segments if the denominators are assumed to be nonzero. Conversely, if the denominators are non-zero and $u_{x_{1} x_{1}} / u_{x_{1} x_{2}}=u_{x_{1} x_{2}} / u_{x_{2} x_{2}}$, then we may define $A$ as in (2), and it follows as explained above that $u$ is affine on line segments that foliate $D$. This proves Theorem 2.5.

\section{Some geometric characterizations of Banach spaces}

To give some background, in order to motivate why we should study at all questions such as those addressed in the theorems of the previous section, we review some literature and results that give rise to questions on biconvex functions. Recall that a function $u: D \rightarrow \mathbb{R}$, defined on a convex subset $D$ of a Banach space, is said to be convex if $u(t x+(1-t) y) \leq$ $t u(x)+(1-t) u(y)$ whenever $x, y \in D$ and $0<t<1$ (note that then $t x+(1-t) y \in D$ ). A function $u: D \times D \rightarrow \mathbb{R}$ is said to be biconvex if for each $x \in D$, the function $y \rightarrow u(x, y)$ is a convex function of $y \in D$, and if for each $y \in D$, the function $x \rightarrow u(x, y)$ is a convex function of $x \in D$. Taken together, the following results should motivate the quest for the best possible biconvex function in the definition of $\zeta$-convexity, for $L^{p}$-spaces.

\section{$3.1 \zeta$-Convexity}

A real or complex Banach space $E$ with norm $\|x\|$ is $\zeta$-convex if there is a biconvex function $\zeta: E \times E \rightarrow \mathbb{R}$ such that $\zeta(0,0)>0$ and

$$
\zeta(x, y) \leq\|x+y\| \quad \text { if }\|x\|=\|y\|=1 .
$$


Clearly the constant function $\zeta \equiv 0$ is biconvex and satisfies (6), so that it is the condition $\zeta(0,0)>0$ that makes the requirements non-trivial. Burkholder has shown that $\zeta(0,0) \leq 1$ and $E$ is a (real or complex) Hilbert space if, and only if, it is possible to have $\zeta(0,0)=1$. Thus for $\zeta$-convex non-Hilbert Banach spaces we have $0<\zeta(0,0)<1$.

It is shown in Lemma 3.1 of [2] that, in order to find the greatest biconvex function $\zeta$ satisfying (6), it is enough to have the function $\zeta$ defined and biconvex on the product of the closed unit ball of $E$ with itself rather than on the whole space $E \times E$.

Let $\bar{B}$ be the closed unit ball in a real Hilbert space $H$ with norm $\|x\|$ and let $\mathcal{F}$ be the class of biconvex functions $u$ on $\bar{B} \times \bar{B}$ satisfying (6). Then we have the following theorem by Burkholder.

Theorem 3.1 (Burkholder [2]) Let $\zeta_{B}$ denote the greatest biconvex function $u$ of $\mathcal{F}$ and let $\langle x, y\rangle$ be the inner product of $x$ and $y$. Then

$$
\zeta_{B}(x, y)=\left(1+2\langle x, y\rangle+\|x\|^{2}\|y\|^{2}\right)^{1 / 2} \quad \text { for all } x, y \in \bar{B}
$$

Note that looking for a maximal $\zeta$ makes sense only when $\zeta$ is restricted to $\bar{B} \times \bar{B}$, since outside this set one could always make $\zeta$ larger without violating any requirements.

\subsection{UMD-unconditional for martingale differences}

Let $\Omega$ be a probability space with a $\sigma$-algebra $\mathcal{A}$ of measurable sets for a measure $P$. A discrete-time $E$-valued martingale $g$ on $\Omega$ is a sequence of $E$-valued functions $g_{n}$ in $L^{1}(\Omega)$ such that $g_{n}$ is measurable with respect to a $\sigma$-algebra $\mathcal{A}_{n}$, where $\mathcal{A}_{n} \subset \mathcal{A}_{n+1} \subset \mathcal{A}$, such that, for each $A \in \mathcal{A}_{n}$, we have $\int_{A}\left(g_{n+1}-g_{n}\right) d P=0$.

The Banach space $E$ is said to be UMD (unconditional for martingale differences) if $\beta_{p}(E)<\infty$ for some $p \in(1, \infty)$; equivalently, for all $p \in(1, \infty)$ by Maurey [5], 1975, where $\beta_{p}(E)$ denotes the least $\beta \in[1, \infty]$ such that

$$
\left\|\sum_{k=0}^{n} \varepsilon_{k} d_{k}\right\|_{p} \leq \beta\left\|\sum_{k=0}^{n} d_{k}\right\|_{p}
$$

for all $E$-valued martingale differences $d_{k}=g_{k+1}-g_{k}$, all sequences $\varepsilon=\left(\varepsilon_{1}, \varepsilon_{2}, \varepsilon_{3}, \ldots\right)$ of numbers in $\{1,-1\}$, and all non-negative integers $n$.

The following theorem shows how the $\zeta$-convexity property characterizes UMD-spaces.

Theorem 3.2 (Burkholder [3]) A Banach space E is UMD if, and only if, it is $\zeta$-convex.

\subsection{HT-space}

Let $1<p<\infty$ and $f: \mathbb{R} \rightarrow E$ belong to $L_{E}^{p}(\mathbb{R})$. For each $\varepsilon>0$, let $H_{\varepsilon}$ denote the truncated Hilbert transform of $f$, that is,

$$
H_{\varepsilon} f(x)=\frac{1}{\pi} \int_{|y|>\varepsilon} \frac{f(x-y)}{y} d y,
$$

where $x \in \mathbb{R}$, and we define $\alpha_{p}(E)$ to be the least $\alpha \in[0, \infty]$ such that

$$
\|H f\|_{p} \leq \alpha_{p}(E)\|f\|_{p} \quad \text { if } 1<p<\infty \text {, where } H f(x)=\lim _{\varepsilon \downarrow 0} H_{\varepsilon} f(x) .
$$


This limit exists for almost all $x \in \mathbb{R}$, see M Riesz [6], 1928.

The Banach space $E$ is said to be an $H T$-space if $\alpha_{p}(E)$ is finite for some $p \in(1, \infty)$; equivalently, for all $p \in(1, \infty)$, see Schwartz [7], 1961 and Benedek, Calderón, and Panzone [8], 1962.

The following theorem shows the relation between HT-spaces and UMD-spaces.

Theorem 3.3 (Bourgain [9], Burkholder [10], McConnell [10]) A Banach space E is an HT-space if, and only if, it is UMD.

By Theorem 3.2 and Theorem 3.3, we obtain the three equivalent conditions giving geometric characterizations of Banach spaces.

\section{Proof of Theorem 2.1}

Let the assumptions of Theorem 2.1 be satisfied. We separate the proof of Theorem 2.1 into two parts. First, we will geometrically prove that $A\left(x_{1}, x_{2}\right)$ is constant on certain line segments that are maximal and then we use this fact to show that $u$ is affine on each such segment.

\subsection{The function $A\left(x_{1}, x_{2}\right)$ is constant on certain line segments}

Let $O=c_{1}+i c_{2}$ be any point of $D$. Set $a=A(O)$. This determines the line $L_{1}$ containing all points $\left(x_{1}, x_{2}\right)=x_{1}+i x_{2}$ such that $\Phi(a)=x_{2}+a x_{1}$, and the point $O$ must be on this line, that is, by assumption, we have $\Phi(a)=c_{2}+a c_{1}$.

Note that any point $\left(x_{1}, x_{2}\right)$ at which $A$ takes the value $a$ must be on line $L_{1}$. Therefore, for each value of $A$, there is a corresponding line segment (the intersection of $D$ and $L_{1}$ ), and the value $a$ cannot be taken anywhere outside that line segment. At this stage there is no guarantee that $A$ cannot take also values other than $a$ on $L_{1}$ since points other than $O$ on $L_{1}$ might lie also on relevant lines other than $L_{1}$.

We seek to prove that $A$ is constant on the intersection of $D$ and $L_{1}$. To get a contradiction, suppose that $A$ is not constant on $D \cap L_{1}$. Then there is a point $P \in D \cap L_{1}$ such that $A(P) \neq a$. We may assume that $A(P)>a$, since the argument that follows would be similar in the case $A(P)<a$.

Write $P=d_{1}+i d_{2}$ and $A(P)=a^{\prime}$. Let $L_{2}$ denote the line of all points $\left(x_{1}, x_{2}\right)$ such that $\Phi\left(a^{\prime}\right)=x_{2}+a^{\prime} x_{1}$. The point $P$ must be on this line, that is, $\Phi\left(a^{\prime}\right)=d_{2}+a^{\prime} d_{1}$. Since $P$ also lies on $L_{1}$, we further have $\Phi(a)=d_{2}+a d_{1}$.

Since $a^{\prime} \neq a$, the lines $L_{1}$ and $L_{2}$ have different slopes and hence intersect at exactly one point, and therefore this point of intersection must be $P$. Note that the point $O$ is not on $L_{2}$.

The line $L_{2}$ intersects the boundary of $D$ at exactly two points, say $P_{1}=a_{1}+i b_{1}$ and $P_{2}=a_{2}+i b_{2}$. Let $L_{3}$ and $L_{4}$ denote the closed line segments from $O$ to $P_{1}$ and from $O$ to $P_{2}$, respectively.

Since $A$ cannot take the value $a$ at any point of $L_{2}$ including $P$, by continuity, all values of $A$ on $L_{2}$ must be greater than $a$ since $A(P)>a$.

Let $b=\min \left\{A\left(P_{1}\right), A\left(P_{2}\right)\right\}$. Since $A\left(P_{1}\right)>a$ and $A\left(P_{2}\right)>a$, we have $b>a$. Since $A$ is realvalued and continuous, $A$ must take all values belonging to the closed interval $\left[a, A\left(P_{1}\right)\right]$ on the segment $L_{3}$. Also $A$ takes all values in the closed interval $\left[a, A\left(P_{2}\right)\right]$ on the segment $L_{4}$. Thus on each of $L_{3}$ and $L_{4}, A$ takes all values on $[a, b]$.

For each value $g \in(a, b)$, there is a point $P^{\prime}$ on $L_{3}$ and a point $P^{\prime \prime}$ on $L_{4}$ such that $A\left(P^{\prime}\right)=$ $A\left(P^{\prime \prime}\right)=g$. Then both $P^{\prime}$ and $P^{\prime \prime}$ must lie on the line of points $\left(x_{1}, x_{2}\right)$ such that $\Phi(g)=$ 
$x_{2}+g x_{1}$. Let this line be denoted by $L_{g}$. Then $L_{g}$ can intersect each of $L_{3}$ and $L_{4}$ only once, and all points where $A$ takes the value $g$ must lie on $L_{g}$.

Concerning values $\alpha<a$ that $A$ might hypothetically take on $L_{3}$, by continuity each such value $\alpha$ would have to be taken at least twice on $L_{3}$. On the other hand, points where the value $\alpha$ are taken must lie on the line $L_{\alpha}$, so that since two distinct points of $L_{3}$ lie on $L_{\alpha}$, it follows that the line $L_{\alpha}$ contains the segment $L_{3}$. This is a contradiction, since the line containing $L_{3}$ must be equal to $L_{\beta}$, where $\beta=A\left(P_{1}\right)>a>\alpha$. Thus $A$ takes no values $<a$ on $L_{3}$. Similarly, $A$ takes no values $<a$ on $L_{4}$. This argument implies that $A$ is one-to-one on each of $L_{3}$ and $L_{4}$. This also means that $P^{\prime}$ and $P^{\prime \prime}$ are unique, for each $g \in(a, b)$. Thus $A$ is strictly increasing on each of $L_{3}$ and $L_{4}$ when we move from $O$ to points where $A$ takes the value $b$.

Consider now values $g>a$ that are arbitrarily close to $a$. Then $P^{\prime}$ and $P^{\prime \prime}$ must also be arbitrarily close to $O$, by the continuity of $A$ and the fact that $A$ is one-to-one on these segments. Moreover, when $g$ is close to $a$, also the line $L_{g}$ must be close to $L_{1}$, and in particular the slope of $L_{g}$, that is, the slope of the line joining $P^{\prime}$ and $P^{\prime \prime}$, must be close to the slope of $L_{1}$.

Since the problem is invariant under translations and rotations when we now discuss only these slopes, we may disregard the connection of the slope to the value $a$, and assume that $O$ is the origin and $L_{1}$ is the real axis. Then $P$ is a non-zero real number and we may assume that $P>0$. The slope of $L_{2}$ is non-zero, and we may assume that the slope is negative since the argument is similar in the other case. If $P_{1}$ is the left end point, then $a_{1}<0$ and $b_{1}>0$, and then $a_{2}>0$ and $b_{2}<0$, see Figure 1 .

Now $L_{3}$ is part of the line $y=-k_{1} x$ and $L_{4}$ is part of the line $y=-k_{2} x$, where $k_{1}=\left|b_{1} / a_{1}\right|$ and $k_{2}=\left|b_{2} / a_{2}\right|$. When $P^{\prime}$ is close to 0 , we have $P^{\prime}=-c+i k_{1} c$ for some small $c>0$, and when $P^{\prime \prime}$ is close to 0 , we have $P^{\prime \prime}=d-i k_{2} d$ for some small $d>0$. The slope of $L_{g}$ is negative, and its absolute value is

$$
\left(k_{1} c+k_{2} d\right) /(c+d) \geq \min \left\{k_{1}, k_{2}\right\}>0
$$

and hence cannot be arbitrarily close to the slope of $L_{1}$, which is zero.

This is the desired contradiction, and it follows that $A$ had to be constant on the intersection of $D$ and the line $L_{1}$. This then shows that the lines $\Phi(a)=x_{2}+a x_{1}$ for various real

Figure 1 An illustration for the proof of Theorem 2.1.

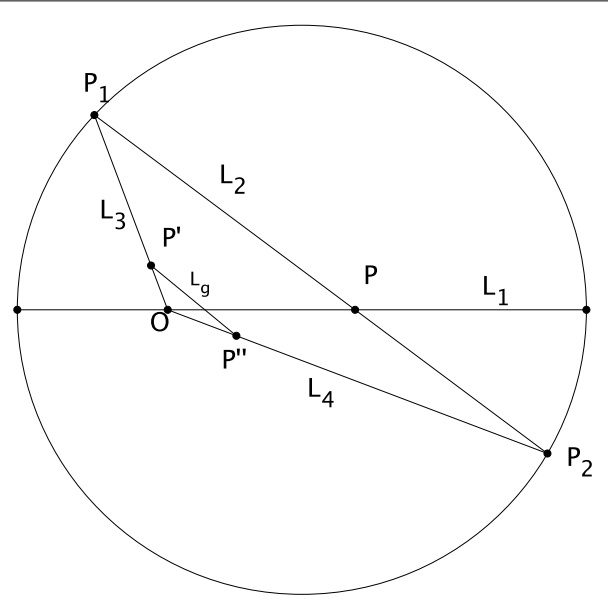


numbers $a$ must foliate $D$, and on each of these lines, the function $A$ takes the constant value $a$. Since a function cannot take two values at the same point, the intersections of these lines with $D$ must be disjoint.

\subsection{The function $u$ is affine on line segments}

Consider a point $\left(x_{1}, x_{2}\right)$ at which $A$ takes a certain value $a$. Set $b=\Phi(a)$. From Section 4.1, we see that $A$ is equal to the constant $a$ on the line $b=x_{2}+a x_{1}$, denoted by $L$. Then $L$ has a unit tangent vector $(c, s)$ where $c=\cos \theta$ and $s=\sin \theta$ for a certain $\theta$, and then $0=s+a c$. We will show that the derivative of $u$ in the direction $(c, s)$ is constant on $L$ so that $u$ is affine on $L$. To see this, we take the derivative of $u$ in the direction of $L$ twice and get

$$
c\left(c u_{x_{1}}+s u_{x_{2}}\right)_{x_{1}}+s\left(c u_{x_{1}}+s u_{x_{2}}\right)_{x_{2}}=c^{2} u_{x_{1} x_{1}}+2 c s u_{x_{1} x_{2}}+s^{2} u_{x_{2} x_{2}} .
$$

On the line $L$, we have $a=A\left(x_{1}, x_{2}\right)=u_{x_{1} x_{1}} / u_{x_{1} x_{2}}=u_{x_{1} x_{2}} / u_{x_{2} x_{2}}$. Hence

$$
u_{x_{1} x_{2}}=a u_{x_{2} x_{2}} \quad \text { and } \quad u_{x_{1} x_{1}}=a u_{x_{1} x_{2}}=a^{2} u_{x_{2} x_{2}} .
$$

Therefore,

$$
\begin{aligned}
c^{2} u_{x_{1} x_{1}}+2 c s u_{x_{1} x_{2}}+s^{2} u_{x_{2} x_{2}} & =c^{2} a^{2} u_{x_{2} x_{2}}+2 c s a u_{x_{2} x_{2}}+s^{2} u_{x_{2} x_{2}} \\
& =(c a+s)^{2} u_{x_{2} x_{2}} \\
& =0 .
\end{aligned}
$$

This completes the proof that $u$ is affine on $L$. The proof of Theorem 2.1 is now complete.

\section{Proof of Theorem 2.3}

Let the assumptions of Theorem 2.3 be satisfied. Thus, let $u$ be a convex function of $\left(x_{1}, x_{2}\right)$ such that $u$ is affine on a certain line segment in the $\left(x_{1}, x_{2}\right)$-plane and let $v=(k, r)$ be a nonzero vector giving the direction at each point of the line segment on which $u$ is affine.

Let $(a, b)$ be a point on a line segment on which $u$ is affine. Then this line segment consists of points of the form

$$
(a+s k, b+s r)
$$

where $s$ is real and runs through an interval of real axis.

On this line segment, $S(s)=u(a+s k, b+s r)$ is a linear function of $s$, which means that $S^{\prime}(s)$ is a constant. We have

$$
S^{\prime}(s)=u_{x_{1}} k+u_{x_{2}} r
$$

where $u_{x_{1}}, u_{x_{2}}$ are evaluated at $(a+s k, b+s r)$.

Therefore, $S^{\prime \prime}(s)=0$ for all $s$ in an interval of real axis, and we have

$$
S^{\prime \prime}(s)=k\left(u_{x_{1}}\right)^{\prime}+r\left(u_{x_{2}}\right)^{\prime},
$$

where $\left(u_{x_{1}}\right)^{\prime}=(d / d s) u_{x_{1}}(a+s k, b+s r)$ and similarly for $u_{x_{2}}$. 
We have

$$
\left(u_{x_{1}}\right)^{\prime}=u_{x_{1} x_{1}} k+u_{x_{1} x_{2}} r
$$

and

$$
\left(u_{x_{2}}\right)^{\prime}=u_{x_{2} x_{1}} k+u_{x_{2} x_{2}} r
$$

where $u_{x_{1} x_{1}}, u_{x_{1} x_{2}}, u_{x_{2} x_{2}}$ are evaluated at $(a+s k, b+s r)$.

Hence

$$
S^{\prime \prime}(s)=k\left(u_{x_{1} x_{1}} k+u_{x_{1} x_{2}} r\right)+r\left(u_{x_{1} x_{2}} k+u_{x_{2} x_{2}} r\right)
$$

so, since $u_{x_{2} x_{1}}=u_{x_{1} x_{2}}$, we have

$$
S^{\prime \prime}(s)=k^{2} u_{x_{1} x_{1}}+2 k r u_{x_{1} x_{2}}+r^{2} u_{x_{2} x_{2}} .
$$

We may write this in the Hessian matrix notation of $u$ as follows.

Let

$$
H=\left(\begin{array}{ll}
u_{x_{1} x_{1}} & u_{x_{1} x_{2}} \\
u_{x_{2} x_{1}} & u_{x_{2} x_{2}}
\end{array}\right) .
$$

By writing vectors generally as column vectors, let

$$
v=\left(\begin{array}{l}
k \\
r
\end{array}\right)
$$

be a column vector and denote its transpose by $v^{T}$. Then we have

$$
S^{\prime \prime}(s)=v^{T} H v=0 .
$$

By the convexity of $u$, we have $\operatorname{det} H \geq 0, u_{x_{1} x_{1}} \geq 0$ and $u_{x_{2} x_{2}} \geq 0$. Hence, $\operatorname{tr} H=u_{x_{1} x_{1}}+$ $u_{x_{2} x_{2}} \geq 0$.

Thus $H$ is a symmetric positive semi-definite matrix, and it has real non-negative eigenvalues. If $H$ is positive definite, that is, if $\operatorname{det} H>0$, then it is well known that $v^{T} H v>0$ for all $v \neq 0$. Thus $\operatorname{det} H=0$, which is equivalent to (4). The last statement of Theorem 2.3 now follows immediately. The proof of Theorem 2.3 is complete.

\section{Conclusion}

We find some structural characterizations of the maximal convex functions $u: D \rightarrow \mathbb{R}$ defined in the closure of a bounded convex plane domain, such that $u(x) \leq \psi(x)$ for all $x \in \partial D$, for a given real-valued function $\psi$ on $\partial D$. This is a more general problem than the one-variable version of finding or characterizing the largest biconvex function $\zeta: \bar{B} \times \bar{B} \rightarrow$ $\mathbb{R}$ such that $\zeta(x, y) \leq|x+y|_{p}$ whenever $|x|_{p}=|y|_{p}=1$, where $\bar{B}$ is the closed unit ball in $\mathbb{R}^{2}$ in the $\ell^{p}$-metric, obtained when the variable $y$ is fixed. We show that if such an extremal 
function is in $C^{3}$, then it is affine on certain line segments that foliate the domain $D$. Thus one can further approach all such problems by specifically looking for such foliations. In 1986, Burkholder explicitly found the maximal function for $p=2$ without the realization of this structural property of this type of extremal functions. This paper provides a greater understanding of the nature of the solutions to these extremal problems.

\section{Competing interests}

The authors declare that they have no competing interests.

\section{Authors' contributions}

The two authors contributed equally to all aspects of the paper, and read and approved the final manuscript.

\section{Author details}

'Department of Mathematics, University of Illinois at Urbana-Champaign, 1409 W Green Street, Urbana, IL 61801, USA.

${ }^{2}$ Department of Mathematics, Faculty of Science, Silpakorn University, Nakhon Pathom, 73000, Thailand.

\section{Acknowledgements}

This material is supported by Faculty of Science, Silpakorn University under the grant SRF-PRG-2558-05.

Received: 4 October 2016 Accepted: 22 November 2016 Published online: 01 December 2016

\section{References}

1. Burkholder, DL: Boundary value problems and sharp inequalities for martingale transforms. Ann. Probab. 12(3), 647-702 (1984)

2. Burkholder, DL: Martingales and Fourier analysis in Banach spaces. In: Probability and Analysis (Varenna, 1985). Lecture Notes in Math., vol. 1206, pp. 61-108. Springer, Berlin (1986). doi:10.1007/BFb0076300

3. Burkholder, DL: A geometrical characterization of Banach spaces in which martingale difference sequences are unconditional. Ann. Probab. 9(6), 997-1011 (1981)

4. Hörmander, L: Lectures on Nonlinear Hyperbolic Differential Equations. Mathématiques \& Applications (Berlin) [Mathematics \& Applications], vol. 26. Springer, Berlin (1997)

5. Maurey, B: Système de Haar. In: Séminaire Maurey-Schwartz 1974-1975: Espaces $L^{P}$, Applications Radonifiantes et Géométrie des Espaces de Banach, Exp. Nos. I et II, pp. 1-26. Centre Math., École Polytech., Paris (1975)

6. Riesz, M: Sur les fonctions conjuguées. Math. Z. 27(1), 218-244 (1928). doi:10.1007/BF01171098

7. Schwartz, J: A remark on inequalities of Calderon-Zygmund type for vector-valued functions. Commun. Pure Appl. Math. 14, 785-799 (1961)

8. Benedek, A, Calderón, A-P, Panzone, R: Convolution operators on Banach space valued functions. Proc. Natl. Acad. Sci. USA 48, 356-365 (1962)

9. Bourgain, J: Some remarks on Banach spaces in which martingale difference sequences are unconditional. Ark. Mat. 21(2), 163-168 (1983). doi:10.1007/BF02384306

10. Burkholder, DL: A geometric condition that implies the existence of certain singular integrals of Banach-space-valued functions. In: Conference on Harmonic Analysis in Honor of Antoni Zygmund (Chicago, III., 1981), vols. I, II. Wadsworth Math. Ser., pp. 270-286. Wadsworth, Belmont (1983)

\section{Submit your manuscript to a SpringerOpen ${ }^{\circ}$ journal and benefit from:}

- Convenient online submission

Rigorous peer review

- Immediate publication on acceptance

- Open access: articles freely available online

- High visibility within the field

- Retaining the copyright to your article 